\title{
QoE-Centric Management of Advanced Multimedia Services
}

\author{
Stefano Petrangeli and Filip De Turck \\ Department of Information Technology (INTEC), Ghent University-iMinds \\ Gaston Crommenlaan 8 (Bus 201), 9050 Ghent, Belgium \\ email: stefano.petrangeli@intec.ugent.be
}

\begin{abstract}
Over the last years, multimedia content has become more prominent than ever. Particularly, video streaming is responsible for more than a half of the total global bandwidth consumption on the Internet. As the original Internet was not designed to deliver such real-time, bandwidth-consuming applications, a serious challenge is posed on how to efficiently provide the best service to the users. This requires a shift in the classical approach used to deliver multimedia content, from a pure Quality of Service (QoS) to a full Quality of Experience (QoE) perspective. While QoS parameters are mainly related to low-level network aspects, the QoE reflects how the end-users perceive a particular multimedia service. As the relationship between QoS parameters and QoE is far from linear, a classical QoS-centric delivery is not able to fully optimize the quality as perceived by the users. This paper provides an overview of the main challenges this $\mathrm{PhD}$ aims to tackle in the field of end-to-end QoE optimization of video streaming services and, more precisely, of HTTP Adaptive Streaming (HAS) solutions, which are quickly becoming the de facto standard for video delivery over the Internet.
\end{abstract}

Keywords: Quality of Experience, Multimedia delivery, Multi-agent algorithms, Adaptive video streaming

\section{Introduction}

Nowadays, multimedia applications are responsible for an important portion of the traffic exchanged over the Internet. As an example, video streaming is responsible for more than a half of the total global bandwidth consumption on the Internet [1]. The continuous growth of these bandwidth-consuming and real-time applications poses a serious challenge on how to efficiently deliver multimedia content over the Internet. As an efficient delivery depends on the quality as perceived by the final user, the so-called Quality of Experience (QoE), a shift is required from a classical Quality of Service (QoS)- to a full QoE-centric delivery. In this perspective, networks and services should be managed in order to guarantee specific QoE levels instead of classical QoS parameters, which are unable to reflect the actual delivered QoE. A pure QoE-centric management is challenged by the nature of the Internet itself, as the Internet was not originally designed to support today's complex and high demanding services. In this PhD we investigate this challenging topic in detail and focus on the following research questions: (i) how cooperation can be envisioned between the network and the 
applications to increase the QoE? (ii) how applications' algorithms can be made adaptive to highly varying network conditions? (iii) how to guarantee fairness from a QoE perspective to applications competing for shared network resources?

\section{QoE-centric delivery of Adaptive Video Streams}

This $\mathrm{PhD}$ research covers the design of an architecture and algorithms for the efficient management of multimedia content. Particularly, we focus on the efficient delivery of HTTP Adaptive video Streaming (HAS).

\subsection{Motivation}

HAS techniques have become very popular due to their flexibility, and can therefore be considered as the de facto standard for video streaming services over the Internet. Recently, a standardized solution has been proposed by MPEG, called Dynamic Adaptive Streaming over HTTP (DASH). In HAS, each video is temporally segmented and stored in different quality levels. Rate adaptation heuristics, deployed at the video player, allow the most appropriate level to be dynamically requested, based on the current network conditions. Nevertheless, several inefficiencies have still to be solved in order to further improve users' QoE. As reported by Akshabi et al., current heuristics perform sub-optimally when sudden bandwidth drops occur, therefore leading to freezes in the video play-out, the main factor influencing users' QoE [2]. This issue is aggravated in case of live events, where the video player buffer has to be kept as small as possible in order to reduce the play-out delay between the user and the live signal. Moreover, current solutions have been shown to be underperforming in a multi-client scenario [3], [4], [5]. Concretely, this means that clients streaming video at the same time negatively influence each other as they compete for shared network resources. Fairness issues are not due to TCP dynamics, but mainly arise from the rate adaptation algorithms, as they decide on the actual rate to download [3]. In this $\mathrm{PhD}$, we aim to solving the aforementioned problems arising in the delivery of HAS streams. Particularly, we focus on two complementary aspects: (i) novel client-based rate adaptation heuristics to improve users' QoE and (ii) network-based systems to efficiently deliver HAS streams.

\subsection{Proposed Approach and Methodology}

As introduced previously, a drawback of current HAS systems is their unmanaged nature. In a classical HAS system, no information exchange is envisioned between the clients and the network nodes. The goal of this $\mathrm{PhD}$ is to reverse this paradigm in order to fully optimize the delivery of HAS streams, i.e., provide a better QoE and improve fairness among competing clients. Particularly, we focus on multi-client scenarios, where multiple clients stream video at the same time. A fundamental aspect to consider in this setting is that the rate adaptation heuristic of a client influences that of the other clients. When a client selects a particular quality level, it uses a portion of the shared bandwidth. This decision has an impact on the performance of the other clients and thus also on their rate adaptation. This means that a group of competing clients creates a distributed control-feedback system, whose performance is difficult to predict and influence. 


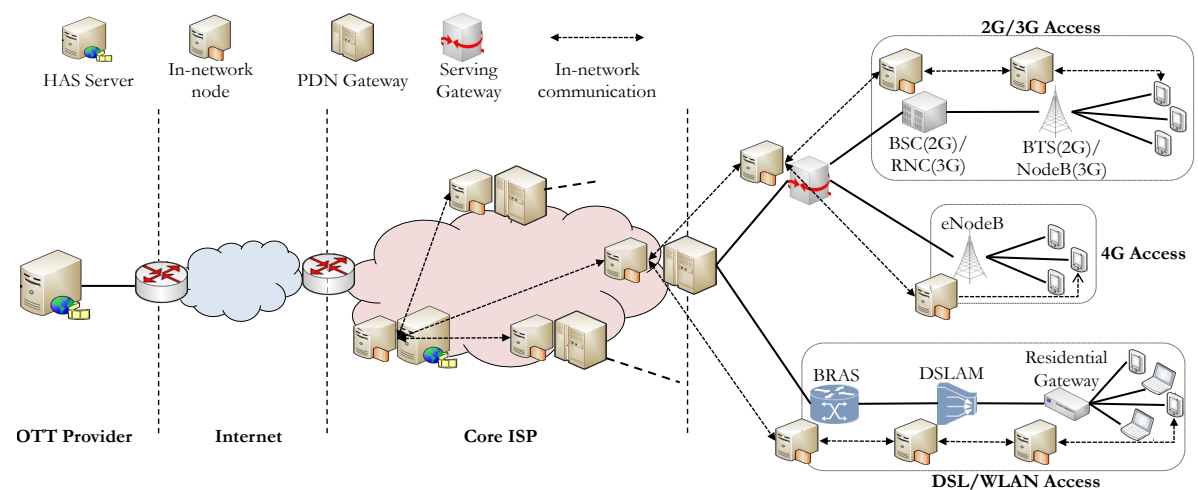

Fig. 1: Schematic representation of a communication network based on the Evolved Packet Core (EPC) [6]. The possible locations of the in-network nodes are shown. A node should be located at the main aggregation points of the network, in order to monitor the links where a bottleneck can occur.

In light of the above, the questions we aim to answering in this $\mathrm{PhD}$ are the following: (i) which feedback can be exchanged by the clients and the network nodes in order to have a fully cognitive delivery chain? (ii) which actions network nodes can take in order to help the clients achieving a high QoE and improving fairness? (iii) how can HAS clients proactively exploit the information provided by the network nodes to enhance the delivered QoE?

In order to tackle these questions, an in-network approach is investigated in this $\mathrm{PhD}$. In this case, in-network nodes are placed in the network to collect feedback regarding the network and clients' conditions and influence client's behaviour. A possible positioning is shown in Figure 1. The main advantage of this approach is three-fold. First, no communication is needed among clients and consequently no significant overhead is introduced. Second, the quality level selection is still performed locally and independently by each client. Third, the approach is robust toward network failures, as the clients can also operate (at a sub-optimal level) without the in-network system. This problem can be modelled as a multi-agent system, where autonomous agents operate on local knowledge and posses only limited abilities, but they are nonetheless able to achieve a desired global behaviour (i.e., increase QoE and fairness). A first step in this area has been taken by us with the implementation of a rate adaptation heuristic based on a multi-agent version of the Q-Learning algorithm [5].

Obtained Results Figure 2a shows some results from our proposal [5]. We investigate the performance of the proposed multi-client HAS framework, in comparison with both the Q-Learning-based client studied by Claeys et al. [7] and a traditional HAS client, the Microsoft ISS Smooth Streaming (MSS) [8], in a scenario with 7 and 10 clients streaming video at the same time. Each bar represents the average QoE of the entire group of clients, together with its standard deviation that we use as fairness metric. The QoE is a metric in the same range of the Mean Opinion Score and can be computed as described by Claeys et al. [7]. In the evaluated bandwidth scenario, we were able to show 


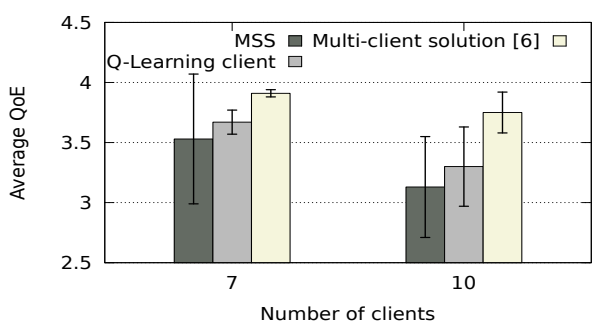

(a)

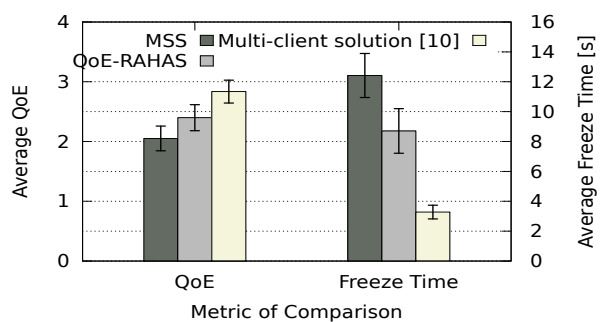

(b)

Fig. 2: Obtained results using the proposed in-network-based approach.

that our multi-client HAS framework resulted in a better video quality and in a remarkable improvement of fairness, up to $60 \%$ and $48 \%$ in the 10 clients case, compared to MSS and the Q-Learning-based client, respectively. In Figure $2 \mathrm{~b}$, a comparison in terms of QoE and freeze time is provided with the MSS, the QoE-RAHAS heuristic [9] and our multi-client solution [10], in a scenario with 30 clients streaming video at the same time. By evaluating our solution under varying network conditions and in several multi-client scenarios, we showed how the proposed approach can reduce freezes up to $75 \%$ when compared to benchmark heuristics.

\section{Conclusions and Future Work}

This $\mathrm{PhD}$ aims to contributing to a more efficient delivery of multimedia content and, more specifically, of HTTP adaptive streams. We have proposed several new HAS heuristics able to dynamically adapt their behaviour depending on network conditions, in order to obtain a high QoE at the client. A fundamental element of our solution is a system of in-network nodes in charge of collecting information on the network and clients' conditions and improve clients' behaviour. Future research proceeds in three directions, which are detailed in the following.

Energy-aware management. Given the consumption of video from mobile devices is constantly increasing, rate adaptation heuristics should become aware and able to adapt to the characteristics of the mobile devices (e.g., CPU, battery lifetime). For this purpose, a MPEG-DASH compliant video player has been developed in order to test the proposed algorithms on real equipment [11].

Heterogeneous clients. In real scenarios, different devices with different characteristics, e.g., in terms of screen dimension or subscription level, stream video together. The quality delivered to each device should be tailored based on these characteristics. This would also allow to introduce new business models in HAS services. How the proposed algorithms should be adapted in order to efficiently tackle this aspect is still an open topic.

High-definition (HD) video delivery. The rapid evolution toward superHD content (e.g., 4K video) poses new challenges in the delivery of multimedia services. As HD content requires bigger HTTP segments, its delivery is much more susceptible to video freezes compared to traditional one. Moreover, the effect of quality switches on QoE is further made worse. We propose to investigate the use of the new HTTP/2 standard to further increase the performance of the proposed algorithms in this scenario. 


\section{Acknowledgement}

This work was partly funded by FLAMINGO, a Network of Excellence project (ICT-318488) supported by the European Commission under its Seventh Framework Programme.

\section{References}

1. Cisco Systems, Cisco Visual Networking Index: Forecast and Methodology, 2012-201\%. [Online]. Available: http://www.cisco.com/c/en/us/solutions/ collateral/service-provider/ip-ngn-ip-next-generation-network/white_ paper_c11-481360.html. Last visited: March 2015.

2. S. Akhshabi, S. Narayanaswamy, A. C. Begen and C. Dovrolis, An Experimental Evaluation of Rate-adaptive Video Players over HTTP. Signal Processing: Image Communication, Volume 27, Issue 4, pp. 271-287. April 2012.

3. S. Akhshabi, L. Anantakrishnan, A. C. Begen, and C. Dovrolis, What Happens when HTTP Adaptive Streaming Players Compete for Bandwidth?. In 22nd International Workshop on Network and Operating System Support for Digital Audio and Video (NOSSDAV '12). 9-14, 2012, ACM, Toronto (Canada).

4. Z. Li, X. Zhu, J. Gahm, R. Pan, H. Hu, A. C. Begen, and D. Oran, Probe and Adapt: Rate Adaptation for HTTP Video Streaming At Scale. IEEE Journal on Selected Areas in Communications (2014), Volume 32, Issue 4, 719-733.

5. S. Petrangeli, M. Claeys, S. Latré, J. Famaey, and F. De Turck, A Multi-agent QLearning-based Framework for Achieving Fairness in HTTP Adaptive Streaming. In 2014 IEEE Network Operations and Management Symposium (NOMS). 1-9, 2014, IEEE, Krakow (Poland).

6. 3rd Generation Partnership Project (3GPP), 3GPP system - fixed broadband access network interworking (3GPP TS 23.139 version 11.3.0 Release 11). [Online]. Available: http://www. 3gpp.org/DynaReport/23139.htm. Last visited: March 2015.

7. M. Claeys, S. Latré, J. Famaey, T. Wu, W. Van Leekwijck, and F. De Turck, Design and Optimization of a (FA)Q-Learning-based HTTP Adaptive Streaming Client. Connection Science 26, 01 (2014), 27-45, Taylor and Francis.

8. A. Zambelli, Microsoft ISS Smooth Streaming (MSS) client. [Online]. Available: https://slextensions.svn.codeplex.com/svn/trunk/SLExtensions/ AdaptiveStreaming. Last visited: March 2015.

9. S. Petrangeli, J. Famaey, M. Claeys, and F. De Turck, A QoE-driven Rate Adaptation Heuristic for Enhanced Adaptive Video Streaming. [Online]. Available: http://users . ugent.be/spetrang/QoE-RAHAS.pdf. Last visited: March 2015.

10. S. Petrangeli, T. Wauters, R. Huysegems, T. Bostoen, and F. De Turck, Networkbased Dynamic Prioritization of HTTP Adaptive Streams to Avoid Video Freezes. In 2015 IFIP/IEEE International Workshop on Quality of Experience Centric Management (QCMan). Ottawa (Canada), May 2015.

11. S. Petrangeli, N. Bouten, E. Dejonghe, J. Famaey, P. Leroux, and Filip De Turck, Design and Evaluation of a DASH-compliant Second Screen Video Player for Live Events in Mobile Scenarios. In 2015 IEEE/IFIP International Symposium on Integrated Network Management (IM). Ottawa (Canada), May 2015. 